\title{
IIdentificación de formas glaciales mediante imagen LANDSAT-7 ETM+ - Costa de fiordos de Patagonia Central, Chile
}

\author{
Rosemary Vieira* \\ Jefferson Cardia Simões* \\ Núcleo de Pesquisas Antárticas e Climáticas - NUPAC \\ IGEO/UFRGS \\ Av. Bento Gonçalves, 9500, prédio 43136, salas 208 e 210 \\ Porto Alegre - RS - Brasil \\ Cep. 91501-970 \\ rosemary.vieira@ufrgs.br
}

\begin{abstract}
RESUMEN
Este trabajo presenta rasgos geomorfológicos identificados e interpretados en imagen Landsat 7 ETM+, de zonas subaéreas adyacentes a los fiordos Peel y Penguin, en la costa de fiordos de Patagonia Central, Chile. Los mapas geomorfológicos tienen el apoyo complementario de bloques diagramas 3D. El retroceso y oscilaciones de los glaciares frente a los fiordos Penguin y Peel ha producido modernos paisajes geomorfológicos que contienen formas glaciales y paraglaciales. La distribución de depósitos glacigénicos subaéreos evidencia control topográfico sobre las líneas de flujo y posibles condiciones térmicas basales húmedas de los glaciares, como los campos de colinas alargadas observados en el margen distal norte del fiordo Penguin. La ubicación de varios tipos de depósitos y rasgos erosionales indica que muchos de los glaciares que hoy están confinados en sus respectivos valles posiblemente se proyectaban sobre los fiordos. Asimismo, los glaciares tributarios retrocedidos en sus respectivos valles alimentan las cuencas de los fiordos mediante flujos glaciofluviales.
\end{abstract}

Palabras Clave: costa de fiordos, campo de hielo, glaciar exutorio, formas glaciales, formas paraglaciales, imágenes Landsat.

\section{Glacial landforms identification by means of LANDSAT-7 ETM+ image - Central Patagonian fjord coast, Chile}

\begin{abstract}
This work presents geomorphological features visually identified and interpreted in Landsat 7 ETM+ images, of subaerial zones adjacent to Penguin and Peel fjords, in the fjord coast of central Patagonia, Chile. Geomorphological maps are made based both on isatellite image and on 3D block-diagrams elaborated by the combination of elevation data and satelital image, thus allowing better visualization. The glaciers retreat produced modern geomorphological landscapes which contain both glacial and paraglacial landforms. The surficial distribution of subaerial glacigenic deposits shows evidences of topographic control on the glacier flow line and possible warm thermal basal conditions of the glaciers, such as fields of elongated hills, observed at the northern distal shoreline of Penguin fjord. The localizations of several kind of deposits and erosional features shows that many of glaciers which, at the present times, are confined in their valleys, possibly reached over the fjords and still contribute the fjord basins with sediment through glaciofluvial flows.
\end{abstract}

Key words: fjord coast, ice field, outlet glacier, glacial landforms, paraglacial landforns, Landsat images. 


\section{INTRODUCCIÓN}

Los seguimientos a los procesos de deglaciación tanto en la Última Glaciación como en el Holoceno han permitido sintetizar el continuum de la deriva glacial en el concepto de paraglacial para los procesos que no son estrictamente glaciales (CURRY \& BALLANTYNE 1992; BALLANTYNE 2002). Grandes sistemas morfológicos glaciales y paraglaciales han podido ser recubiertos gracias al análisis de imágenes satelitales (p. ej. ABER et al. 1993), principalmente debido a las dificultades de acceso a terreno en las regiones donde estos sistemas tienen mucha representación a escala regional. Imágenes satelitales como las Landsat 7 ETM+ (Enhanced Thematic Mapper Plus) y ASTER (Advanced Spaceborne Thermal Emission and Reflection Radiometer) de resolución espacial de 30 y $15 \mathrm{~m}$, respectivamente, pueden usarse con ventaja para estos fines. Las imágenes CBERS-2 (China-Brazil Earth Resources Satellite) proveen resolución espacial de hasta $5 \mathrm{~m}$. Con estos materiales han podido aplicarse modelos digitales de elevación (SMITH et al. 2006).

En la costa de fiordos de Patagonia norte y central, ha sido posible identificar formas recientes de deglaciación en las zonas de las cabeceras y en los valles de fiordos (GLASSER et al. 2005; VIEIRA 2006). En las zonas adyacentes a los campos de hielo Norte y Sur las áreas recientemente deglaciadas exhiben rasgos morfológicos, aún no afectados definitivamente por acciones paraglaciales que han podido ser identificadas por la combinación de bandas de imágenes ETM+. Desde los años 90 las imágenes satelitales de la serie Landsat han sido aplicadas en la identificación de rasgos glaciales en diversos ambientes (e.g. CLARCK 1993; ABER et al. 1993; PUNKARI 1995; CLAPPERTON \& SUGDEN 1998; KLEIN \& ISACKS 1997; HARRISON \& WINCHESTER 1998 y 2000), donde las morrenas preservadas y otros rasgos geomorfologicos marcan las extensiones máximas de los glaciares.
Al conocimiento sobre formas glaciales subaéreas en Patagonia Central, se puede agregar las observaciones sobre geomorfología submarina de fiordos y canales de la región. Aquí, ARAYA-VERGARA (1999) encontró bancos morrénicos y explanadas de represamiento, que pueden representar dos fases de estacionamiento de los glaciares en recesión: la Fase Externa y la Fase Interna de los Fiordos. Así, ARAYAVERGARA (2003) pudo establecer que los fiordos de Patagonia Central constituyen un tipo caracterizado por contener bancos morrénicos y explanadas de represamiento. Por consiguiente, pueden servir de referencia para interpretar las probables formas glaciales sub-aéreas.

Este trabajo tiene como propósito mostrar formas glaciales en las zonas de deglaciación del Campo de Hielo Patagónico Sur - junto a las cabeceras y a los valles de los fiordos - y ampliar el conocimiento sobre su significado, emplazamiento y repartición regional.

\section{MATERIALES Y MÉTODOS}

\section{Área de estudio}

El área de estudio cubre parte del dominio interno de la costa de fiordos chilena, en la Patagonia Central. Fueron seleccionadas dos áreas: una que abarca el fiordo Penguin y otra que corresponde al fiordo Peel, incluyendo también los fiordos Calvo y Amalia. Las cabeceras de los fiordos en esta zona son alimentadas por glaciares del tipo tidewater que fluyen desde el Campo de Hielo Patagónico Sur (Fig. 1).

La topografía de esa área es dominada por la Cordillera de los Andes que forma un eje de campos de hielo que se extienden por más de $1.800 \mathrm{~km}$, entre $38^{\circ} \mathrm{S}$ e $56^{\circ} \mathrm{S}$. El Campo de Hielo Patagónico Sur es el más grande en zonas templadas en el hemisferio sur y la segunda masa de hielo en el hemisferio tras la Antártica, con extensión total de $350 \mathrm{~km}$ y anchura media de $35 \mathrm{~km}$, constituyendo un área de $\sim 13.000 \mathrm{~km} 2$ (CASASSA et al. 2000). Está formado por 48 grandes glaciares y por 


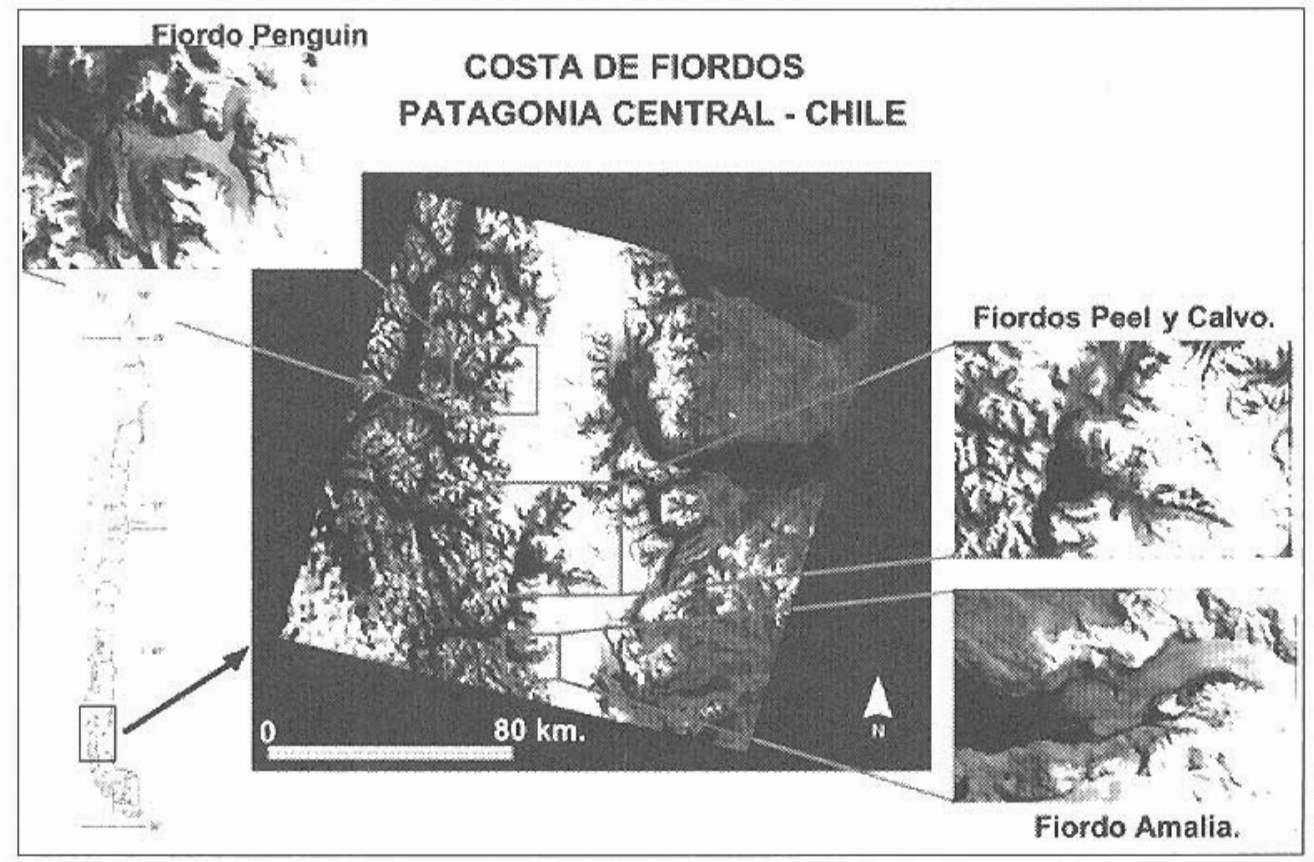

Fig. 1. Localización del área de estudio.

Fig. 1. Location of studied area.

más de un centenar de pequeños glaciares de circo y de valle. Los glaciares exhutorios asociados, generalmente, tienen terminales desprendentes (calving glaciers) en lagos al Este y en fiordos al Oeste (ANIYA et al. 1996 y 1997; CASSASA et al. 2000). Estos glaciares están unidos a la zona de acumulación del campo de hielo, que constituye un plateau, en general plano, en su sector mas elevado y una altitud media de $1.600 \mathrm{~m}$.

El campo de hielo Patagónico Sur se conecta con el océano Pacífico a través de una compleja red de canales y fiordos que presenta rasgos similares a los de otras costas de fiordos. Esta red se extiende en la práctica por toda Patagonia Occidental.

Actualmente, se observa un retroceso generalizado de los glaciares en 42 casos del campo de hielo (ANIYA et al. 1997), mientras cuatro glaciares se encontraban estables entre 1944 y 1986 (HPS 13, HPS 15, Calvo y Spegazzini), dos avanzaron en el mismo periodo (Pío XI y Moreno). El principal retroceso se produjo en el glaciar O'Higgins, con pérdidas de $0,48 \mathrm{~km}$ a-1 de la zona frontal, entre el verano de 1944-45 y 1985-86 (ANIYA et al. 1997). En el mismo periodo, el mismo glaciar retrocedió $14,6 \mathrm{~km}$ (CASASSA et al. 1997). El avance máximo fue observado en el glaciar Pío XI con una tasa de 1,45 km2 a-1 entre 1946-1986 y un avance frontal de $0,29 \mathrm{~km} \mathrm{a-1}$ (RIVERA et al. 1997). Sin embargo, desde 1997, dicho glaciar también está retrocediendo.

\section{Procedimientos}

Para este estudio, se usó el producto del sensor de alta resolución espectral Enhanced Thematic Mapper Plus (ETM+) montado en el satélite LANDSAT-7. La imagen posee una resolución espacial de $30 \mathrm{~m}$ y siete bandas espectrales adquiridas en 14-10-2001 (Row: 095; Path: 231; coordenadas centrales: Lat. 50.2836630 , Long, $-73.4431891^{\circ}$ ).

La imagen original LANDSAT-7 ETM+ está georeferenciada bajo el sistema de coordenada UTM/WGS 84. El programa ERDAS IMAGINE 8.7 fue utilizado para (re) muestreo y rectificación geométrica de la imagen. Un mínimo de 20 puntos de control 
(GCPs) de rasgos identificables fue colectado en mapas 50.000 (fiordo Penguin) y 70.000 (fiordo Penguin) del Servicio Hidrográfico y Oceanográfico de la Armada de Chile (SHOA), con una transformación polinomial de primer-grado y (re) muestreo del vecino más cercano (nearest neighbour resampling). El error RMS (Root-Mean Square) fue 30 pixels.

Aunque la Patagonia Central es un área de elevada precipitacion y nubosidad, la imagen analizada está libre de cobertura de nubes. Para la interpretación de la geomorfología glacial de la parte subaérea de las cuencas adyacentes a los fiordos fue utilizada la combinación de las bandas 1,4 y 5 , de resolución espacial de $30 \mathrm{~m}$. Su combinación provee un mejor contraste entre hielo, roca y vegetación, posibilitando de esta manera buena cobertura del área subaérea alrededor del campo de hielo y de los fiordos (GLASSER et al. (2005). Además, en esas áreas los procesos de denudación no han actuado efectivamente sobre las formas debido a los recientes procesos de deglaciación. La identificación de las formas y su subsiguiente mapeo se baso en GLASSER \& JANSSON (2005), GLASSER et al. (2005), SMITH et al. (2006) y GUSTAVSSON et al. (2006). Las formas glaciales identificadas en las imágenes fueron digitalizadas en pantalla con el software ARCVIEW 3.2. Con el uso de softwares comerciales GLOBAL MAPPER 6 fue posible crear diagramas en 3D reproduciendo parte de la morfología subaérea. La interpolación y elaboración de datos de elevación fueron hechas mediante el método Topogrid del programa ArcInfo 8.0.2.

\section{RESULTADOS}

\section{Formas glaciales subaéreas}

Los glaciares del tipo tidewater ocupan las cabeceras de los fiordos en el área de estudio (Fig. 1). Los que llegan a las cabeceras de los fiordos fluyen desde el campo de hielo, estando la mayoría confinada dentro de los valles.
Hacia la cabecera del fiordo Penguin, que está dividida en dos brazos, fluyen por lo menos dos glaciares (Fig. 1 y Fig. 4, diagrama «b»). Lo mismo ocurre en la del fiordo Peel (Fig. 5 , diagrama «a»). Con respeto al fiordo Calvo, aparte del glaciar del mismo nombre, que baja directamente al fiordo en la forma de cascada de hielo, varios otros drenan el campo de hielo a través de sus bordes laterales (Figs. 1 y 5, diagramas «b» y «c»). Algunos de los glaciares de valle que alcanzan las cabeceras del fiordo Peel y el glaciar Amalia están cubiertos en extensiones variadas por detritos supraglaciales, los cuales tienen las mismas características espectrales de las áreas adyacentes (Fig. 5, diagrama «e»). El delineamiento de las cubiertas por detritos fue hecho manualmente. Los límites de las lenguas de hielo cubiertas pueden estar formados por una morrena lateral o por las paredes laterales de los valles, pero éstas no fueron distinguidas en el trabajo (Fig. 5, diagrama «d»).

Diversas formas son visibles en las zonas proglaciales subaéreas, reflejando las fluctuaciones de los glaciares, posiblemente, durante el Holoceno. En el área de estudio fueron identificados siete tipos principales de formas glaciales (Figs. 2 y 3 ).

En los actuales glaciares de base terrestre existen morrenas terminales, a lo largo de los valles circundantes de los fiordos y también en lugares en donde no hay en el presente lenguas glaciales. Gran parte de las morrenas identificadas ya poseen cobertura vegetal, lo que puede deberse a cierta antigüedad de esas formas o también a la elevada humedad en la costa de fiordos. Los cordones morrénicos, muchos de ellos separados por lagunas terminales, se extienden en algunos lugares por más de $5 \mathrm{~km}$ de la línea de costa hasta los terminales glaciales (Figs. 3 y 5, diagramas «c» $\mathrm{y}$ «d»). Por otro lado, el flujo continuado de sedimentos de los glaciares tributarios retrocedidos desarrolló zonas fluviales y fluviodeltaicas, como las identificadas en la Fig. 4, diagramas a, f y g. 


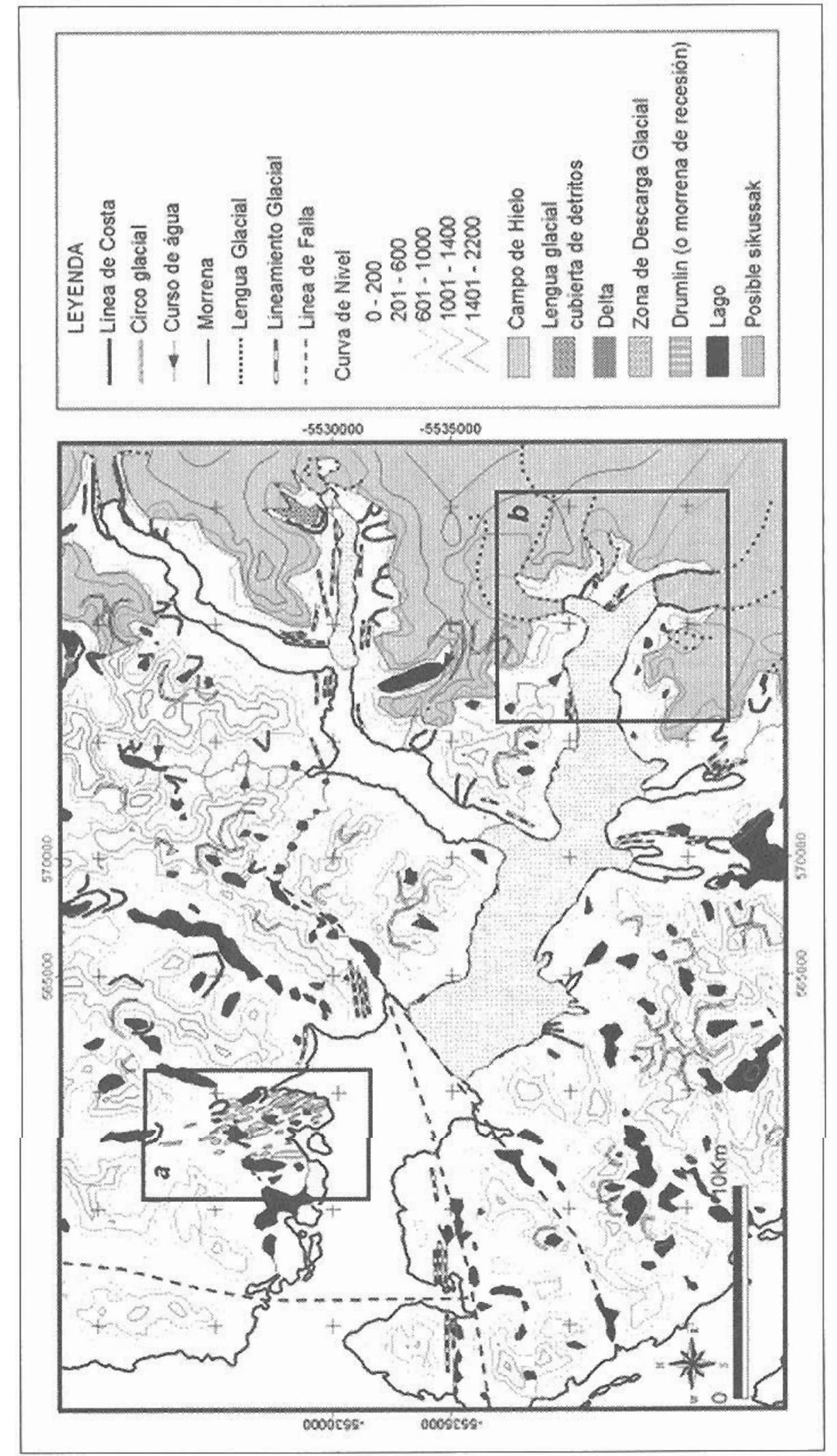

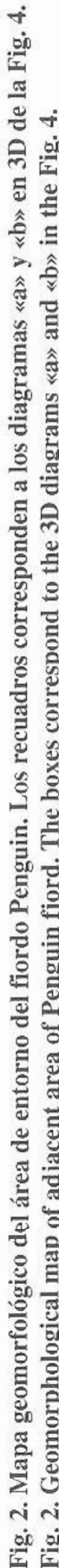



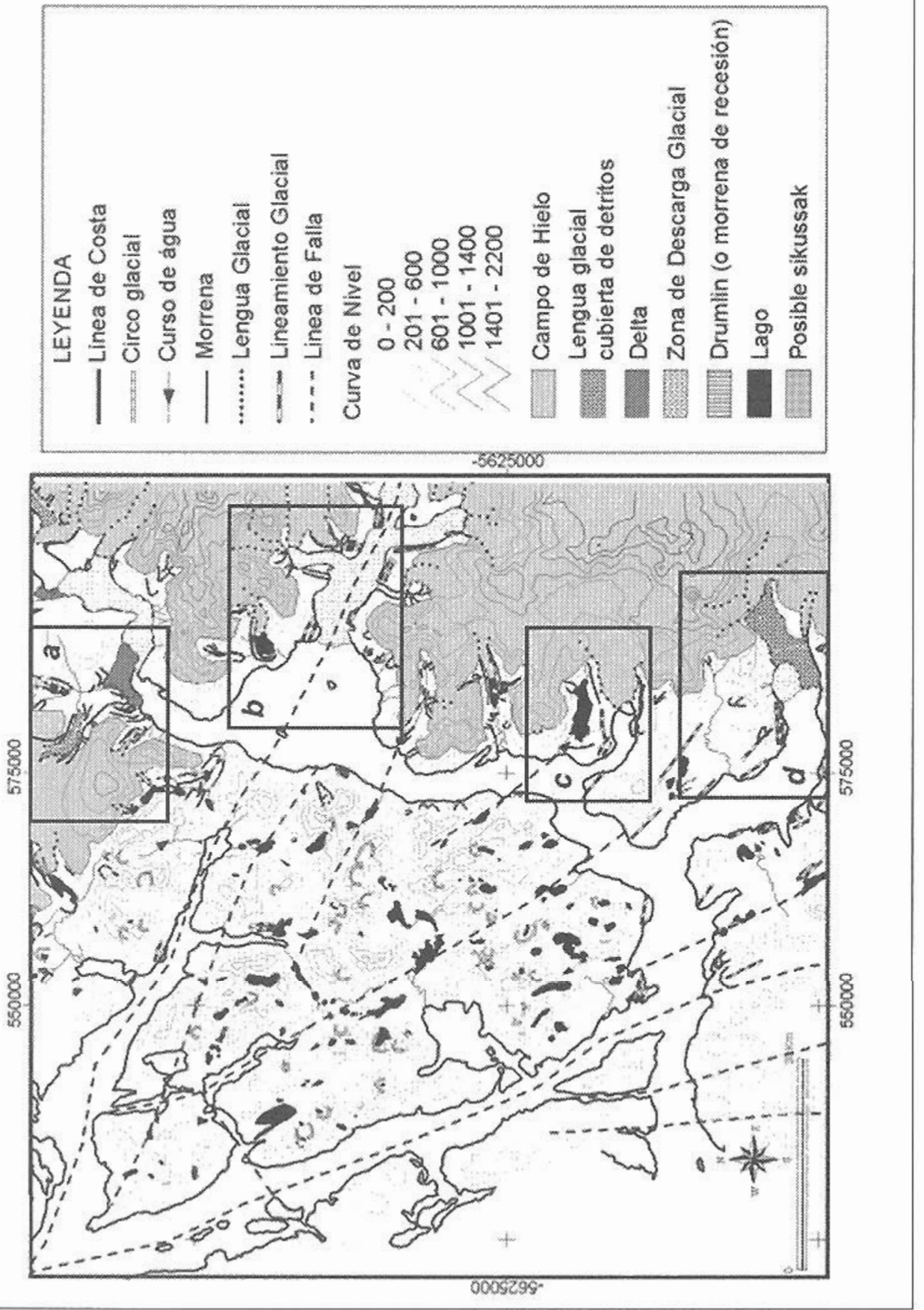


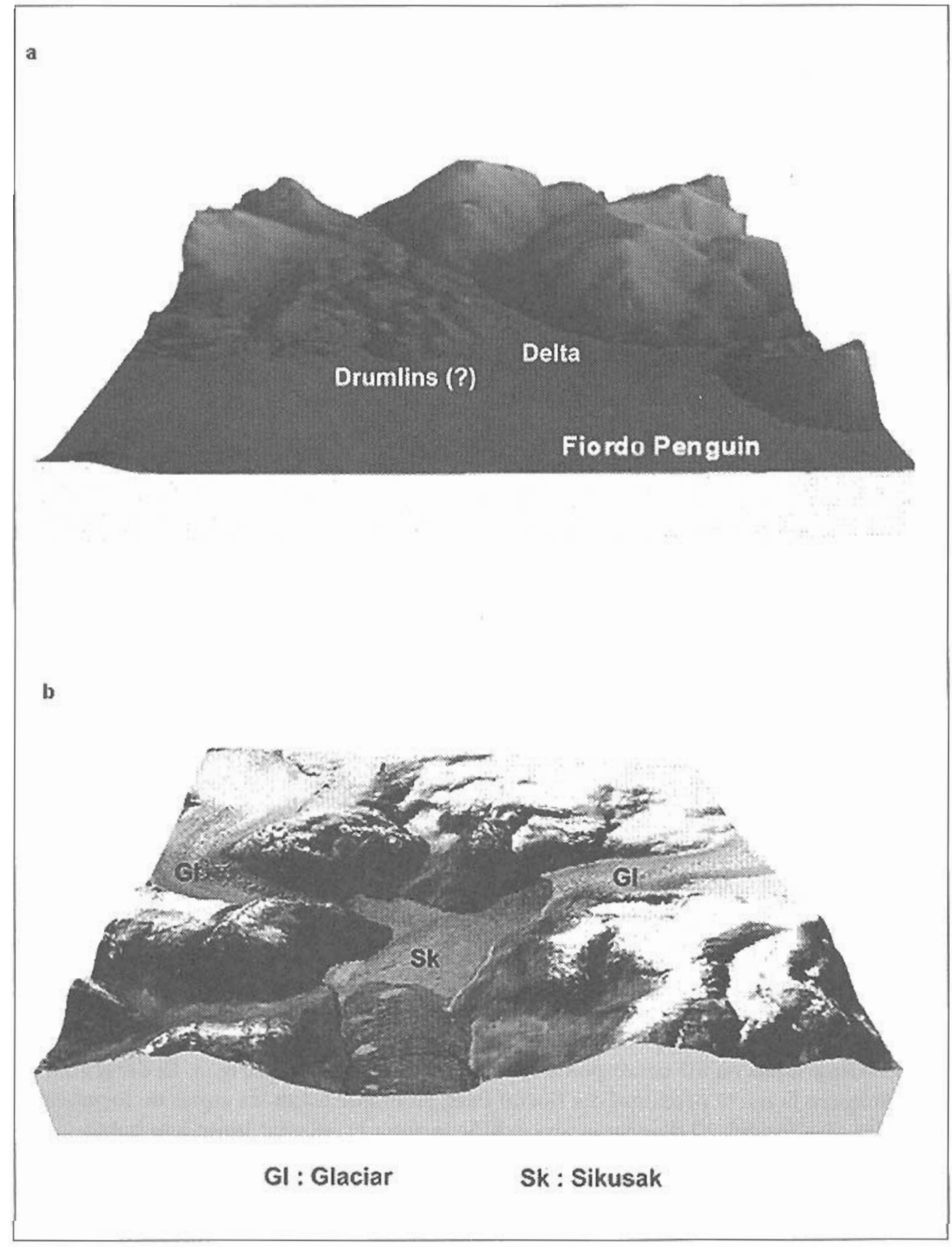

Fig. 4. Diagramas en 3D correspondientes a los recuadros de la Fig. 2. EI diagrama «a» representa el campo de drumlins (o morrenas de recesión) en una zona de delta, en la parte centro-distal del fiordo Penguin. El diagrama «b» representa la zona de cabecera del fiordo, donde se encuentran dos frentes de glaciar tipo tidewater.

Fig. 4. 3D diagrams which correspond to the Fig. 2 boxes. The diagram «a» represents the drumlins field in the central-distal part of Penguin fjord. The diagram «b» represents the fjord head zone with two tidewater glacier fronts. 


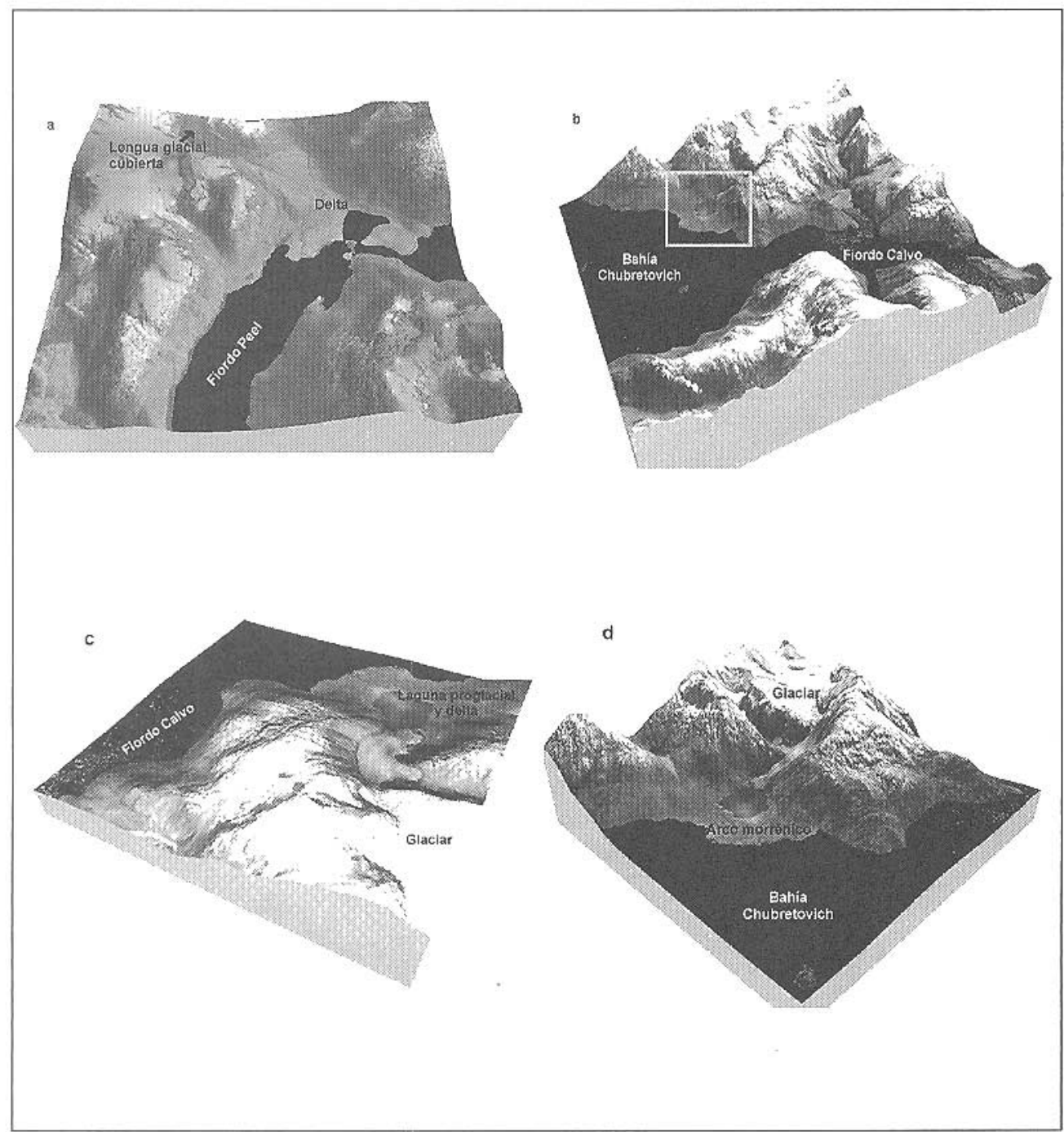

Fig. 5. Diagramas en 3D correspondientes a los recuadros de la Fig. 3. El diagrama «a» muestra la parte proximal del fiordo Peel, en donde están las zonas de formación de delta glacifluvial. El diagrama «b» exhibe la zona proglacial junto a la bahía Chubretovich, en donde se nota la laguna proglacial cercada en su parte frontal por un arco morrénico. Los diagramas «c»y «d» son escenas más aproximadas del mismo sector.

Fig. 5. 3D diagrams which correspond to the Fig. 3 boxes. Diagram «a» shows the proximal area of Peel fjord, with development of glacifluvial delta. The diagram «b» shows the proglacial zone next to Chubretovich bay, in which a proglacial lake is surrounded by morainic belt. The diagrams $\langle\mathrm{c} »$ and $« \mathrm{~d} »$ are special views of the same sector. 


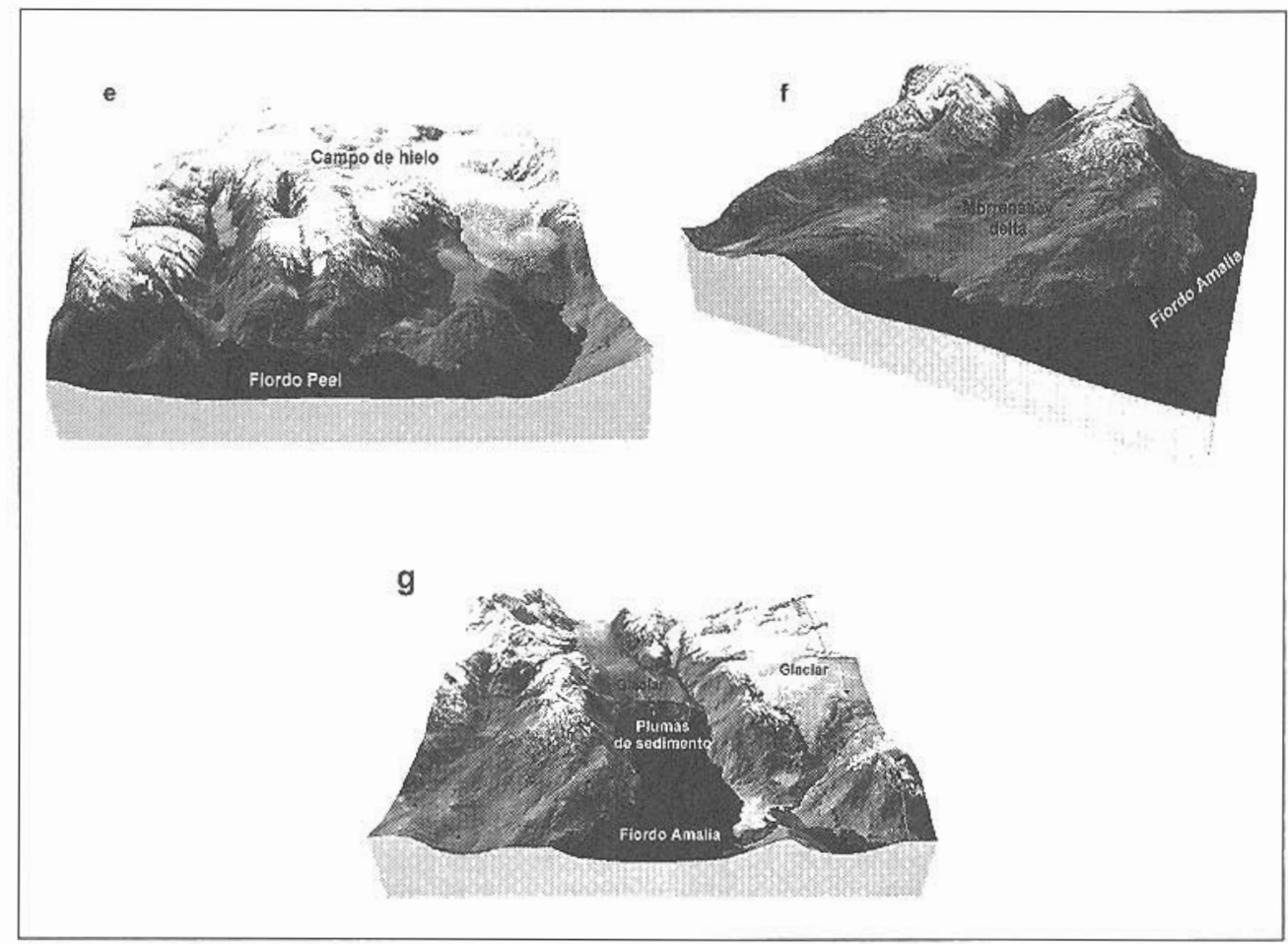

Fig. 5-cont. El diagrama «e» ilustra valles deglaciados y la presencia de lagunas proglaciales. Los diagramas «f» $\mathbf{y}$ «g» corresponden al fiordo Amalia: «f» muestra un valle deglaciado con morrenas y formación de deltas en el sector norte del fiordo en la confluencia del fiorde Peel; «g» muestra el frente del glaciar con gran cantidad de plumas de sedimentos, lo que puede representar actividad de fusión subglacial, es decir, en la base submarina del glaciar.

Fig 5-cont. Diagram «e» ilustrate deglacial valleys with proglacial lakes. Diagrams «f» and $\langle\mathrm{g} »$ indicate sectors of Amalia fjord: «f» shows a deglacial valley with moraines and deltas at the confluence of fjord Peel; «g» shows plumes of sediment at the glacier front, which may represent subglacial melting activity at the submarine glacier base.

Campos de lomas longitudinales alargadas se observan principalmente en el margen norte del fiordo Penguin, en su parte distal, ocupando una área aproximada de $17 \mathrm{~km} 2$, y con cerca de $7 \mathrm{~km}$ de extensión desde la línea de costa hasta el arco morrénico (Fig. 4, diagrama a). Por la localización de estas lomas se infiere un flujo glacial desde el norte, posiblemente de base termal húmeda, coalescente en ese sector del fiordo. Según experiencias de terreno en Magallanes, sólo el trabajo de campo permite decidir si se trata de drumlins o de morrenas de recesión longitudinales, porque se trata de formas parecidas, pero genéticamente muy distintas ( Prof. J.F. Araya Vergara, Universidad de Chile, comunicación verbal). Esto, porque los drumlins y flutes son indicativos de degradación diferencial infraglacial en la dirección del flujo glacial (KLEMAN 1994), mientras que las morrenas recesionales longitudinales son formas deposicionales de descarga.

Extensas áreas con lineamientos glaciales sobre superficies rocosas son visibles en las imágenes de satélite, y están concentradas a lo largo del eje de los fiordos principales y tributarios. Dichos rasgos geomorfológicos 
son generalmente asociados a los proceso de abrasión de las rocas y están alineados con la ruta de las descargas más importantes del hielo. Los ejemplos más notables están en los fiordos Peel y Calvo. La dirección de esos lineamentos y la localización de los depósitos morrénicos en la Fig. 3 indican que el flujo de hielo convergía para la Bahía Chubretovich.

Algunos lineamientos glaciales, superficies de abrasión y un gran número de lagos pueden verse en zonas más elevadas (interfluvios). No obstante, dichos lineamientos se concentran a lo largo del eje principal de cada valle e indican los caminos principales del hielo. Seguramente, la concentración de estas formas es mayor en el área más próxima al campo de hielo que en las áreas más externas y, en algunas de esas superficies, la altitud alcanza a $800 \mathrm{~m}$. La presencia de lagos en esa altura está también asociada a las superficies de abrasión.

Junto a los terminales de los glaciares que drenan a los fiordos Penguin, Peel y Calvo hay desprendimiento de témpanos. Es probable que, debido a la posible formacion de hielo marino se formen sikussaks, una masa aparentemente sólida de icebergs, bergy bits y de hielo marino (SYVITSKI et al. 1996) (Fig. 3 y Fig. 4, diagrama «b»). Los sikussaks parecen formarse cuando las estrecheces del fiordo (pinning points) o umbrales submarinos atrapan los icebergs desprendidos de los glaciares, dificultando de esa manera la circulación de ellos a lo largo de los fiordos y alterando el patrón de sedimentación en ellos. El fiordo Calvo, debido a su configuración y a la confluencia de muchos glaciares, puede contener sikussak. Sin embargo, la presencia actual de este rasgo en estos fiordos es conjetural, puesto que las imágenes no permiten captar bien el fenómeno, para el que el indicador básico es la presencia de hielo marino (Prof. J.F. Araya Vergara, Universidad de Chile, comunicación verbal). Por otro lado, el terminal del fiordo Amalia indica actividad subglacial, visible mediante las plumas de agua de fusión en el sector sur de su terminal (Fig. 5, diagrama «g»).

\section{DISCUSIÓN}

El retroceso de los glaciares a lo largo del eje principal de los fiordos está relacionado con los cambios en el ambiente terminal. La observación actual puede centrarse en tres aspectos: glaciares de cabecera de fiordo con desprendimiento de hielo (calving glaciers), glaciares terrestres y formas glacigénicas que representan transiciones temporales entre esos dos tipos de emplazamiento. Se asume que los cambios asociados tienen influencia morfogenética y ambiental importante sobre los fiordos da Patagonia Central.

En primer lugar, glaciares subaéreos continuan enviando sedimentos terrígenos a las cuencas submarinas vía sistemas de flujos proglaciales. Por otro lado, con la teledetección de áreas de abrasión de rocas, lineamentos glaciales y formas glacigénicas se ha podido avanzar en el conocimiento de los límites y dirección de los glaciales subaéreos, infiriendo el trayecto de la recesión desde el medio marino al terrestre. Para aproximaciones geocronológicas y morfogenéticas de estadios de recesión, los trabajos de MERCER (1968 y 1970) en valles que enfrentan fiordos de la región y compilaciones de CLAPPERTON (1983) son muy útiles (Prof. J.F. Araya Vergara, Universidad de Chile, comunicación personal). Aunque se carece de perfiles acústicos transversales a los fiordos que pudieran mostrar depósitos próximos a sus paredes laterales, es posible inferir, basado en los registros acústicos submarinos (ARAYA-VERGARA 1999; VIEIRA 2006), aporte de sedimentos de origen paraglacial a las capas espesas de sedimentos atrapados en las diversas cuencas submarinas distribuidas a lo largo de los fiordos. No obstante, lo mạs importante en éstos es la distribución de los bancos morrénicos, hecho que es importante comparar con los resultados de MERCER (1968 y 1970), según comunicación personal del Prof. J.F. Araya Vergara (Universidad de Chile.

Por su parte, la distribución de depósitos glacigénicos subaéreos evidencia un control 
topográfico sobre las líneas de flujo, al parecer por condiciones térmicas basales húmedas de los glaciares (SUGDEN \& JOHN 1976). Los campos de colinas alargadas observados en el margen distal norte del fiordo Penguin (Fig. 3, diagrama a), pueden reflejar este hecho. En áreas al Este del campo de hielo Patagónico Norte, GLASSER \& JANSSON (2005) interpretan la presencia conjetural de drumlins y flutes como resultante del flujo rápido del hielo. Como aquí, los lineamientos glaciales en las zonas subaéreas adyacentes a los fiordos de Patagonia Central muestran antiguas líneas de flujos. La diferencia consiste en que, en esta área, los glaciares bajan abruptamente al nivel de mar, formando glaciares del tipo tidewater, o dependiendo de la profundidad, lenguas de hielo. En cambio al norte (Campo de Hielo Patagónico Norte) fue posible incluso la formación de glaciares de piedmont (GLASSER et al. 2005).

\section{CONCLUSIÓN}

La evolución del paisaje en respuesta al retroceso de los glaciares alrededor de los fiordos Penguin y Peel ha producido modernas superficies geomorfológicas observadas mediante imagen satelital Lansat 7 - ETM+. Glaciares aún fluyen por las cabeceras de los fiordos observados. Sin embargo, los glaciares tributarios retrocedidos en sus respectivos valles alimentan las cuencas de los fiordos vía flujos glaciofluviales. La ubicación de varios tipos de depósitos y rasgos erosionales evidencia que muchos de los glaciares que hoy están confinados en sus respectivos valles posiblemente se proyectaban sobre los fiordos. Flujos proglaciales desde cada glaciar han producido zonas fluvio-deltaicas en las zonas distales de los vales tributarios.

Un paisaje paraglacial ha producido flujo de sedimentos hacia los fiordos, incluyendo los resultantes de la erosión de los ríos y de los glaciares, lo que puede ayudar a explicar las espesas capas de sedimentos en las cuencas submarinas de los fiordos analizados, además de los importantes aportes desde los bancos morrénicos de descarga encontrados por (ARAYA VERGARA 1999). Futuros estudios de datación de los depósitos identificados pueden precisar la escala temporal de los eventos glaciológicos.

\section{REFERENCIAS}

ABER, J.S., E.E. SPELLMAN \& M.P. WEBTER, 1993. Landsat Remote Sensing of Glacial Terrain. In: Aber, J.S (Ed.), Glaciotectonics and Mapping Glacial Deposits: Proceedings of he INQUA Comission on Formation and Properties of Glacial Deposits. Canadian Plains Research Center, University of Regina, Regina, pp. 215-225.

ANIYA, M., H. SATO, R. NARUSE, P. SKVARCA \& G. CASASSA, 1996. The use of satellite and airborne imagery to inventory outlet glacier of Southern Patagonia Icefield, South America. Photogramm. Eng. Remote Sens., 62: 1361-1369.

ANIYA, M., H. SATO, R. NARUSE, P. SKVARCA \& G. CASASSA, 1997. Recent variations in the Southern Patagonia Icefield, South America. Arct., Alp. Res, 29: 1-12.

ARAYA-VERGARA, J.F., 1999. Perfiles longitudinales de fiordos de Patagonia Central. Cien. Tecn. Mar, 22: 3-29.

ARAYA-VERGARA, J.F., 2003. Tipología y clasificación de fiordos y piedmonts submarinos de Magallanes, Chile. Invest. Geogr., Chile, 37: 21-40.

BALLANTYNE, C.K., 2002. Paraglacial geomorphology. Quaternary Sci. Rev., 21: 1935-2017.

CASASSA, G., H. BRECHER, A. RIVERA \& M. ANIYA, 1997. A century-long record of glacier O’Higgins, Patagonia. Ann. Glaciol., 24: 106-110.

CASASSA, G., A. RIVERA, M. ANIYA \& R. NARUSE, 2000. Características glaciológicas del Campo de Hielo Patagónico 
Sur. Ann. Inst. Patag., Serie Ciencias Naturales, 28: 5-22.

CLAPPERTON, C.M., 1983. The glaciation of the Andes. Quatern. Sci. R., 2: 83-155

CLAPPERTON, C.M. \& D.E. SUGDEN, 1998. Holocene glacier fluctuations in South America and Antarctica. Quaternary Sci. Rev., 7: 185-198.

CLARCK, C.D., 1993. Mega-scale glacial lineations and cros-cutting ice-flow landforms. Earth Surf. Process. Landforms., 18: 1-29.

CURRY, A.M. \& C.K. BALLANTYNE, 1999. Paraglacial modification of glacigenic sediment. Geogr. Ann., 81 A: 409-419.

GLASSER, N.F. \& K.N. JANSSON, 2005. Fast-flowing outlet glacier of the Last Glacial Maximum Patagonian Icefield. Quaternary. Res., 63: 206-211.

GLASSER, N.F., K.N. JANSSON, S. HARRISON \& A. RIVERA, 2005. Geomorphological evidence for variations of the North Patagonian Icefield during the Holocene. Geomorphology, 71: 263-277.

GUSTAVSSON, A., E. KOLSTRUP \& A.C. SEIJMONSBERGEN, 2006. A new symboland-GIS detailed geomorphological mapping system: Renewal of a scientific discipline for understanding landscape development. Geomorphology, en prensa.

HARRISON, S. \& V. WINCHESTER, 1998. Historical fluctuations of the Gualas and Reicher glaciers, North Patagonian Icefield, Chile. The Holocene, 8: 481-485.

HARRISON, S. \& V. WINCHESTER, 2000. Nineteenth- and twentiethcentury glacier fluctuations and climatic implications in the Arco and Colonia Valleys, Hielo Patagonico Norte, Chile. Arct., Antarc., Alp. Res., 32: 5563.

KLEIN, A.G. \& B.L. ISACKS, 1997. Alpine glacial geomorphological studies in the central Andes using Landsat thematic mapper images. Glacial Geology and Geomorphology, Rp01/1998. http://ggg.qub.ac.uk/ggg/papers/ full/19987rp011998/rp01..htm.

KLEMAN, J., 1994. Preservation of landforms under ice sheet and ice caps. Geomorphology, 9: 19-32.

MERCER, J.H., 1968. Variation of some Patagonian glaciers since the Late glacial: I. Amer. J. of Sci., 266: 91-109.

MERCER, J.H., 1970. Variation of some Patagonian glaciers since the Late glacial: II. Amer. J. of Sci., 269: 1-25.

PUNKARI, M., 1995. Glacial flow systems in the zone of confluence between the Scandinavian and Novaya Zemlya ice sheets. Quatenary Sci. R., 14: 589-603.

RIVERA, A., J. ARAVENA \& G. CASASSA, 1997. Recent fluctuations of glaciar Pío XI, Patagonia: discussion of glacial surge hypothesis. Mount. Res. Develop., 17 (4): 309-322.

SMITH, M.J., J. ROSE \& S. BOOTH, 2006. Geomorphological mapping of glacial landforms from remotely sensed data: An evaluation of the principal data sources and an assessment of their quality. Geomorphology, 76: 148-165.

SUGDEN, D.E. \& B. JOHN, 1976. Glaciers and Landscape. Edward Arnold, London, 376 pp.

SYVITSKI, J.P.M., J.T. ANDREWS \& J.A. DOWDESWELL, 1996. Sediment deposition in an iceberg-dominated glacimarine environment, east Greenland: basin fill implications. Global. Plan. Change, 12, 251-270.

VIEIRA, R., 2006. Interpretação integrada de sísmica de alta-resolução e da morfologia submarina da costa de fiordes da Patagônia Central - Chile. Tese de Doutorado. Instituto de Geociências, Universidade Federal do Rio Grande do Sul, Porto Alegre, $165 \mathrm{pp}$. 